\title{
LINE PROFILES OF FACULAE AND PORES
}

Edward N. Frazier

The Aerospace Corporation

\section{INTRODUCTION}

Historically, attempts to model the temperature structure of faculae have generally suffered from a rather basic contradiction. Models which were based on center to limb measurements of the continuum contrast of faculae disagree with models that are based on measurements of line profiles in faculae. The "continuum" models predict line weakenings which are of larger amplitude than what is observed, and the "line profile" models predict a continuum contrast that is less than what is observed. Chapman (1976) discusses this problem in some detail. It is the purpose of this paper to show that there is a fundamental reason for this historical contradiction between line profile measurements and continuum contrast measurements: The line profile and the continuum contrast of a given facular are both a function (the two functions are different) of the size of that facula. The first indication of this fact was given by Frazier (1971). Figure 1 shows the contrast of faculae in the core of the line Fe I $\lambda 525.0 \mathrm{~nm}$, and in the continuum, as a function of the observed magnetic flux. One can see immediately that the contrast in each channel depends on $\Phi$ in a much different manner. Therefore, one can conclude that the shape of the entire line profile will vary as a function of $\Phi$. On the basis of Figure 1, we must expect that this va riation of the line profile will be continuous from infinitesimally small faculae up through very large faculae, and indeed, all the way up to pores.

We now wish to show that, for any given spectral line, there exists an entire family of facular and pore line profiles. We will show examples of this family of line profiles for two spectral lines; Fe I $\lambda$ $525.0 \mathrm{~nm}$ and $\mathrm{Fe} I \lambda 524.7 \mathrm{~nm}$. It will then become evident that such an entire family must be observed in order to provide an adequate set of data on the temperature structure of faculae and pores. From the data that is available so far, it appears that this family can be characterized by a single parameter. The best parameter to use for this purpose is the amount of magnetic flux, $\Phi$, contained within a facula. At first thought, it might seem that the magnetic field strength, $B$, is the best 

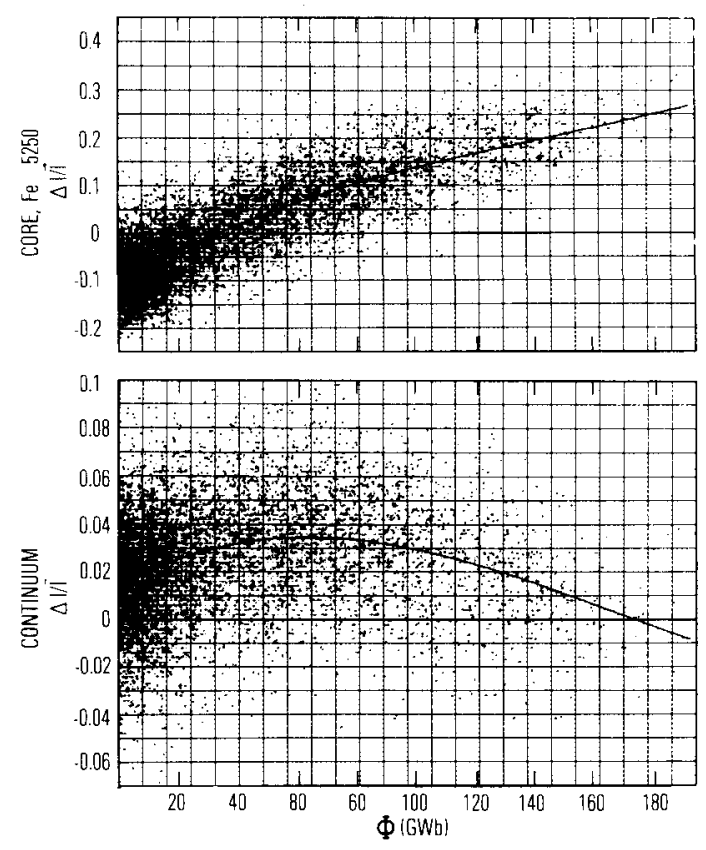

Figure 1. Facular contrast in the core of a temperature sensitive line $(\mathrm{Fe} \lambda 525.0 \mathrm{~nm})$ and in the continuum as a function of observed magnetic flux. In this figure only, the contrast is defined as $\left(I_{f}-I\right) / I$, where $I$ is the average brightness of the region scanned. Adapted from Frazier (1971).

parameter, but it has been shown by Frazier and Stenflo (1972) that the magnetic field strength (the entire distribution of field strength) is the same for all faculae. Therefore, "strong" faculae differ from "weak" faculae by virtue of the total magnetic flux they contain, not the field strength. The area of a facula changes in accordance with the flux. From this point of view then, the terms, "strong" or "weak" faculae should be replaced by "large" or "small" faculae. The radius of a facula would be an equivalent parameter to use instead of the magnetic flux. However, the magnetic flux can be easily measured by a magnetograph, and the radius cannot be easily measured, because it is usually less than the resolution element of the telescope.

Therefore, the task is to observe a family of line profiles (including the continuum), with each profile identified by its corresponding magnetic flux. Operationally speaking, there are two different ways in which one can obtain this data: One can either take the spectrum (plus a magnetic measurement) of one facula after another until a large number of faculae have been observed, or else one can scan a large 
a rea (i.e., many faculae) at a set position within a line profile $(\Delta \lambda)$, then change the $\Delta \lambda$, repeat the scan, and so on until many $\Delta \lambda^{\prime}$ s have been observed. Both techniques yield a brightness measurement as a function of $\Delta \lambda$ and $\Phi$. Since the faculae and pores are being identified only by their magnetic flux, and not by e.g. their location, or their age, the two different techniques produce equivalent data. The second technique is, however, much easier, because it is easily adapted to the operation of a magnetograph. The present observations use this second technique.

\section{THE OBSER VATIONS}

All the observations were made with the Kitt Peak multi-channel magnetograph. The same pair of lines were used in this study as were used earlier by Stenflo (1973); Fe I $\lambda 525.022 \mathrm{~nm}$ and Fe I $524.706 \mathrm{~nm}$. Four different exit slit geometries were chosen to sample the line profile. The exit slits were always $2.5 \mathrm{pm}$ wide, and were centered successively at $\Delta \lambda=2.25,4.75,7.25$ and $9.75 \mathrm{pm}$ from the line center. Four separate raster scans were made of each region that was observed. The only instrumental parameter that was changed between the four scans was the position of the exit slits. The magnetograph was calibrated every time the exit slit positions were changed. The scanning aperture was always $2.4 \times 2.4 \mathrm{arc} \mathrm{sec}$, and the size of the raster scans were usually $160 \times 160$ arc sec.

The observations were made during the period of May 4, 1974 through May 11, 1974. For each absorption line, 4 quantites were recorded; the longitudinal component of the magnetic field and of the velocity, the brightness in the wing, and the brightness in the core. Additionally, the continuum brightness was recorded. Both the magnetic channels and the velocity channels were calibrated by standard techniques, using a drift scan across the disk of the sun. In the subsequent data reduction, the magnetic channels were calibrated in units of giga Webers $(\mathrm{GWb})$. The brightness channels were not calibrated in terms of the disk center continuum intensity at the time the observations were made. So all the brightness measurements were simply transformed to contrast units, $\Delta I / I_{p h}$, where $I_{p h}$ is the brightness in
neighboring non-magnetic regions.

\section{STATISTICAL CONTRAST PROFILES}

For each available brightness channel of each raster scan, a scatter plot was made of the observed contrast as a function of the observed magnetic flux. Such scatter plots are similar to that shown in Figure 1. These points were then averaged over $10 \mathrm{GWb}$ intervals of the observed magnetic flux. This results in a series of tables which contain the observed contrast as a function of $\Delta \lambda$ and $\Phi$. 
One correction must be made to these tables. The observed flux at a given $\Delta \lambda$, say $\Delta \lambda=2.25 \mathrm{pm}$, is less than the observed flux at another $\Delta \lambda$, say $\Delta \lambda=7.25 \mathrm{pm}$ because, when the magnetograph exit slits are set at the narrower $\Delta \lambda$, the instrument suffers from more Zeeman saturation. It would be best to evaluate the total Zeeman saturation for each exit slit setting $(\Delta \lambda)$ and to correct the observed magnetic flux at that $\Delta \lambda$ to the true magnetic flux. However, this would require the use of a specific facular model to calculate the distribution of field strength of faculae, and it is desired to keep this data model-independent. There is a way to correct for the differential Zeeman saturation between the various $\Delta \lambda^{\prime} s$ in a completely empirical manner. This allows us to place all of the data on a common scale of magnetic flux which is internally self-consistent, but which may be different from the true magnetic flux due to Zeeman saturation. This empirical flux correction procedure is based on the fact that the same continuum contrast of faculae is always measured for each different exit slit setting. Each scan then produces one curve of continuum contrast as a function of observed magnetic flux, (for example, Figure 1b) and all such curves should be identical to each other. The differential Zeeman saturation is revealed by a change in the horizontal scale for different $\Delta \lambda^{\prime} \mathrm{s}$. It is a simple matter then to renormalize these flux scales to the flux scale from a chosen standard $\Delta \lambda . \Delta \lambda=4.75 \mathrm{pm}$ was the chosen standard.

With this correction, one has the desired family of line profiles. These profiles are somewhat unorthodox in that the observed points in any given profile do not come from a single feature. Instead, each point is an average measurement over many features, all containing the same amount of magnetic flux. For this reason, these profiles might best be termed "statistical contrast profiles". The relatively low spectral resolution $(2.5 \mathrm{pm})$ is the result of using a magnetograph to measure the profiles instead of a spectrometer. This is a disadvantage which one must contend with in return for the advantage of a simultaneous magnetic flux measurement at every point.

One of the regions scanned near the center of the disk had a significantly higher proportion of large faculae and pores, so the results from that region are presented in Figure 3. Large faculae were observed in sufficient number up to a flux level of about $150 \mathrm{GWb}$.

\section{CONCLUSION}

The single principal conclusion of this paper is obvious from Figure 2. The shape of a line profile, and therefore the $T(\tau)$ relation, of faculae changes siginficantly and continuously from small faculae to pores. This conclusion carries with it two very important implications. The first implication is that it should be possible to calculate a generalized model of a "magnetic feature" which explicitly contains the magnetic flux as the fundamental parameter. This model would then be capable of reproducing the appearance of both faculae and pores just by changing the amount of flux. 


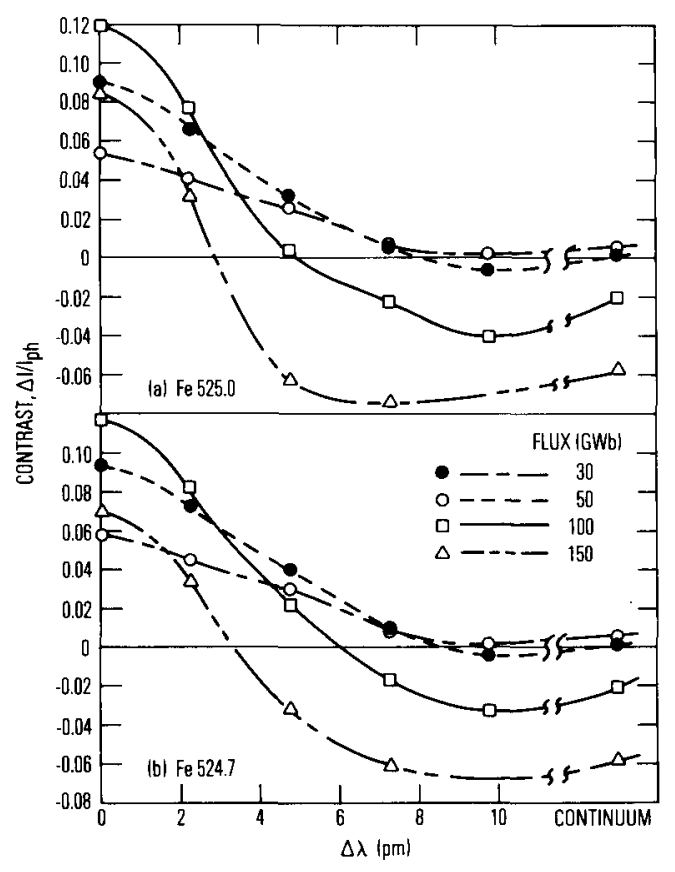

Figure 2. Observed statistical contrast of faculae and pores. These observations were made of an active region at $\mu=.91$. The contrast profiles are labelled by the observed facular

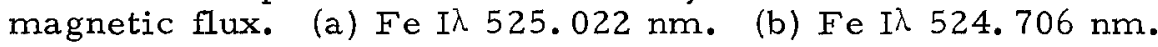

The second implication is that past observations of line profiles of faculae have suffered from observations selection. In making facular observations, it has generally been necessary to perform some kind of a seach procedure first (e.g. search for the brightest $H \alpha$ plage, search for the highest intensity in the core of the desired line, etc.). The very fact that a search process is used means that one member of this famicly of profiles has been selected preferentially. If different observations have used different search techniques, the selection effects will also be different, and it will be very difficult to compare the observations with each other, or with any given model. For example, continuum contrast observations tend to select those faculae which are bright in the continuum (small $\Phi$ ), whereas line profile observations tend to select those faculae which are bright in the cores of lines (large $\Phi)$. Therefore models based on these two different types of observations refer to different types of faculae, and they should not be expected to agree with each other. 


\section{ACKNOWLEDGEMENTS}

The data reported in this paper was obtained at Kitt Peak National Observatory. The author wishes to express his appreciation to $\mathrm{Dr}$. J. Harvey and Mr. B. Gillespie for their invaluable as sistance in operating the McMath telescope and the multi-channel magnetograph. Extensive programming assistance was given by Mrs. T. Becker. This project was supported by The Aerospace Corporation companysponsored research program.

\section{REFER EN CES}

Chapman, G. A.: 1976, Astro. Phys. J., in press. Frazier, E. N.: 1971, Solar Phys. 21, 42. Frazier, E. N. and Stenflo, J. O.: 1972, Solar Phys. 27, 330. Simon, G. W. and Weiss, N. O.: 1971, Solar Phys. 13, 85. Stenflo, J. O.: 1973, Solar Phys. 32, 41. 